\section{Lessons of the "Pink Spot"}

There is nothing like a snappy name for catching the public imagination. The demonstration of the "pink spot" in schizophrenia fired the enthusiasm of journalists, accountants, and scientists in other fields in a way the name dimethoxyphenylethylamine could never do.

The spots which caused the excitement were not on the patients but on paper chromatograms of extracts from their urines, and became visible and coloured only after special staining. The very simplicity of the name conveyed (quite falsely) that the observations and interpretations were simple, in welcome contrast to the greater part of modern science. In fact this work was done in a biochemical-psychiatric borderland where very few people know enough to feel really at home. ' Few biochemists know much about psychiatry. Few psychiatrists feel other than extremely modest about their knowledge of biochemistry. Consequently each kind of specialist has his doubts about a new claim from the borderland, but feels not quite enough of an overall expert to express them. Nevertheless, the consensus of opinion at a recent meeting of psychiatrists at the Royal Society of Medicine was that the "pink spot" is now dead. It remains to say what went wrong and to see what is left.

Human urine contains substances as the Milky Way has stars. They are many and various, a few obvious and striking ; many are mere traces. Frequently special methods are needed for their isolation and identification. What is found in a urine specimen depends, therefore, on how much urine is tested, how fresh it is, how much the specimen can be concentrated, and how sensitive a test can be applied to the concentrate. It also depends on a large number of ordinary physiological variables-such as the degree of diuresis, alkalinity of the urine, time of day it was collected, and the diet of the patient.

A. J. Friedhoff and E. van Winkle ${ }^{1}$ took a 20 -hour specimen of urine from 19 schizophrenic patients and 10 normal people. They made the urine alkaline to $p H 9$ and shook it with ethylene dichloride, which is a standard way of extracting basic substances of organic type from urine-such as tryptamine and other amines which are formed by the body, and many drugs and their metabolites. They concentrated this extract to a very small volume so that a single drop put on the starting line at one end of a paper strip represented about two hours' urine production. The drop was allowed to dry, and the paper strip held vertically and dipped into a butanolacetic-acid-water mixture. This solvent rose slowly up in the paper to the top, drawing with it from the starting line at different speeds the different ingredients of the urine concentrate. Thus the ingredients were dispersed vertically in line up the paper. When the paper was dry and stained with ninhydrin a number of them showed up as blue spots. After overstaining with Ehrlich's aldehyde reagent the blue spots faded, but a single pink spot often stood out about two-thirds the way up the paper. This pink spot was present in 15 schizophrenic patients, but in none of the normal people. The

'Friedhoff, A. J., and Van Winkle, E., f. nerv. ment. Dis., 1962, 135 ,

I 550 . Harper, P., and Leslie, S. A., Nature (Lond.), 208, 453.

s Friedhoff, A.' J., and Van Winkle, E., f. chromatogr., 1963, 11, 272. Williams, C. H., Gibson, J.
(Lond.), 1966, 211, 1195. (Lond.), 1966, 211, 1195.

- Kuehl, F. A., jun., et al., ibid., 1964, 203, 154. 606.

Wagner, A. F., Cirillo, V. J., Meisinger, M. A. P., Ormond, R. E., Kuehl, F. A., and Brink, N. G., ibid., 1966, $211,604$.

- Kety, S. S., Science, 1959, 129, 1528, 1590. authors thought that it might be an amine hitherto unrecorded in human urine-dimethoxyphenylethylamine (D.M.P.E.)and that it might have some connexion with schizophrenia. Subsequently workers in Liverpool applied the same technique to several hundred schizophrenics and normal persons, and confirmed the presence of a single pink spot in a large number of the former. These findings looked uncommonly interesting:

Nevertheless, there are several difficulties in interpreting them. A single pink spot is not necessarily a single substance, or even the same substance every time the test is done. The position of any spot on a paper chromatogram means very little, because it is apt to get displaced by miscellaneous substances in the urinary extract, which vary in nature and do not take up the stain. A pink colour is given by many substances with an ethylamine side-chain, not just D.M.P.E. ${ }^{3}$ Moreover, one-way paper chromatography is too simple a technique for analysis, particularly if used after a simple extraction of the original urine. Thus when a further simple analytical step is introduced-a second chromatographic run at right angles to the first before the ninhydrin staining-the " pink spot" is sometimes broken up into two or more spots, but in any case the spot separates from D.M.P.E. if this has been added to the extract deliberately. ${ }^{4}$ Everybody excretes several amines in the urine in small amounts-and these are increased if they have been taking a drug of the monoamine oxidase inhibitor group up to three weeks beforehand. This type of extraction procedure will display a dozen or so natural amines ; some may come from the diet, some from the bowel, some are the natural products of metabolism.

More elaborate techniques, including ion-exchange resin chromatography and gas chromatography, are now being used in these investigations. One group of workers has already shown that small amounts of D.M.P.E. and of dimethoxyphenylacetic acid can be found in the urine of some schizophrenic patients and of some normal persons, and that these probably originate in the food. ${ }^{56}$ A further finding, with radioactive dihydroxyphenylalanine (D.O.P.A.), was that this normal brain metabolite does not give rise to D.M.P.E. either in vivo or in vitro." On the biochemical side, then, the "pink spot" has vanished, and its place has been taken by the general problems of amine metabolism.

Readers of the cautionary review by S. S. Kety, ${ }^{8}$ which describes a number of biochemical downfalls in the past, will note that schizophrenics may drink more coffee than nurses, or get less vitamin $\mathrm{C}$, or smoke more. They may take more patent medicines on the side-laxatives, pep-pills, cough lozenges-or use less toothpaste. They are often less cleanly in their habits. Their visitors may give them sweets and extras not noted officially by the hospital. Some patients sit or stand a lot, others have a low fluid intake, and many more differences from normal subjects could be instanced. All these matters are reflected ultimately in the composition of the urine. Hence virtually no biochemical abnormality which is found in the urine of a psychotic is likely to have any aetiological importance ; it is overwhelmingly likely to be secondary to some quirk of the patient's behaviour. Moreover, if any substance is to be investigated for its role in schizophrenia it must be subjected to the most rigorous set of ward controls, however tedious it is to impose them and to confirm their exact action. The "pink spot" would fail on this clinical approach, even if it survived the laboratory one.

Many psychiatrists believe that the only feature common to all schizophrenics is a disorder of logical thinking, which may 
be very subtle and hard to detect, and there is a vast literature on the "group of schizophrenias," to employ Bleuler's original use of the word he invented. In some types of schizophrenia hallucinations are a prominent symptom; in other types they are never observed. Some show periods of stupor or excitement; others never have any disorder of bodily movement control. Some have periods of illness with spontaneous remission; others have a continuous illness. It is hard for a clinician to believe that all these different clinical types will prove to be a biochemical unity; indeed there is already a kind of biochemical test which seems to prove the contrary-only some schizophrenics improve with phenothiazine treatment. Diagnostic categories such as epilepsy do not imply that all cases are due to one cause. These categories are, nevertheless, useful, and that of schizophrenia is no exception. It is also hard for a clinician to accept any theory of aetiology which does not explain why the disorder is limited to certain parts of the brain. The majority of schizophrenic patients have no bodily abnormalities, they are physically fit, and even their nervous systems function completely normally except in one or two specialized respects. It must be a very subtle, localized, biochemical anomaly which will account for a schizophrenic illness.

One way in which the findings about the "pink spot" appealed to biochemists was that dimethoxyphenylethylamine is chemically close to trimethoxyphenylethylamine, or mescaline, which is well known for its hallucinogenic properties. Nevertheless, D.M.P.E. is not itself appreciably active in this way, while hallucinations are not a characteristic symptom of the schizophrenias but are found also in epilepsy, depressive illness, and some confusional states.

Speculations of this kind are valuable for the way in which they stimulate enthusiasm and new experimental work and ideas. Nevertheless, perhaps the time has now finally come to stop investigating "schizophrenics" en masse, and to concentrate on individuals. This way, with modern specialized techniques, and the wariness Kety ${ }^{8}$ has suggested, may enable workers eventually to strike biochemical gold.

\section{Prevention of Bedsores}

Often painful, and a possible source of cross-infection, bedsores can be a serious complication for an ill or enfeebled patient to bear. They lead to loss of protein and provide a culture medium for pathogenic bacteria. These may be coliform organisms from the patient's bowel. More dangerous are antibiotic-resistant staphylococci, while Pseudomonas pyocyanea may spread from the sores to the respiratory tract of other old people in a ward, with fatal consequences. Moreover, pressure sores greatly increase the amount of nursing that must be devoted to a patient. Certainly it is an error to regard them lightly or as the inevitable accompaniment of terminal illness.

It is normally impossible to change a patient's position every half hour, in addition to all the other nursing care that may be required, without a nurse-patient ratio which can be provided only in exceptional circumstances. But the avoidance of prolonged pressure on any one part of the body can be achieved by the use of a special mattress. One such, the large-celled ripple mattress, is reported on by Dr. A. N. Exton-Smith and his colleagues at page 394 of the B.M.F. this week. It is a development of the small-celled ripple mattress with narrow cells 2 in. $(5 \mathrm{~cm}$.) wide when deflated. Experience with the latter has shown that it is not entirely satisfactory in providing effective alternating pressure. In the large-celled mattress the cells are 6 in. $(15 \mathrm{~cm}$.) wide.

The mattress is built up of a number of cells or bags running transversely; they extend from the patient's shoulders to below his heels. Alternate cells are connected together to make two groups. By means of an electrical air pump each group of bags is alternately inflated and deflated so that the patient is supported for five minutes on one set of bags and five minutes on the other. As a result no part of the superficial tissues is compressed between the patient and the bed for longer than five minutes. Dr. Exton-Smith and his colleagues have shown that this large-celled ripple mattress is highly effective in preventing bedsores and will hasten healing when sores are present.

Now that an effective means of prevention is described, it is important to predict which patients are especially liable to bedsores. As aids to assessing the danger Dr. Exton-Smith and colleagues suggest that the physician should consider the patient's general condition, the mental state, the degree of activity, the degree of mobility, and the degree of incontinence. He gives the patient marks from 0 to 3 in each of these five categories, so that, for example, continence scores 0 , an occasional accident scores 1 , usually incontinent of urine scores 2 , and double incontinence scores 3 . The worst mark possible is therefore 15 . These authors found that only $4 \%$ of patients with scores less than 2 developed sores and that $51 \%$ of patients with scores of 9 or over suffered them. They suggest that a score of 7 or over represents a substantial danger but emphasize that a rising score is especially ominous.

Thus a doctor should immediately be on the alert and take measures against the development of bedsores when treating comatose patients and those with hemiplegia or other paralysis. Very obese patients and others who do not move easily are also vulnerable, as well as those who are too frail and weak or cannot move because of breathlessness. Such patients are at considerable risk even with a score index under 7.

When the ripple mattress is in use no treatment should be given to the pressure points except to include them in the general washing and powdering of the skin. If a sore is present treatment consists of a simple non-adherent dry dressing. Established infection is best treated by local antibiotics, supplemented by systemic antibiotics if there is cellulitis round the sore. Weak hypochlorite compresses are of value in accelerating the separation of sloughs. When being nursed on the mattress, patients should not be sat up for long periods at a time lest their backs be damaged by pressure from the chair, particularly if they tend to slide forward. An alternating-pressure cushion would be an advantage for those patients who should not be nursed lying flat and might well be worth developing.

Ripple mattresses can be made available under the National Health Service. They take their power from the mains supply and are simple to use. It has been said that they are unreliable, suffering from leaking and failure of the pumps. However, with any apparatus care is needed to see that it is functioning properly. It is important to test them frequently by sliding a hand beneath the patient along the channel produced by a collapsed bag, making sure the body $i_{i}$ clear of the bed, and checking that the bags are in fact alternating. Care must be taken to protect the tubes from 